\title{
Hydrogen Storage in Microporous Hypercrosslinked Organic Polymer Networks
}

Colin D. Wood, Bien Tan, Abbie Trewin, Hongjun Niu, Darren Bradshaw, Matthew J. Rosseinsky, Yaroslav Z. Khimyak, Neil L. Campbell, Ralph Kirk, Ev Stöckel and Andrew I. Cooper*

Department of Chemistry, The University of Liverpool, Crown Street, Liverpool, L69 3BX, United Kingdom.

Supporting Information 




Figure S1. Plot of $\mathrm{H}_{2}$ uptake at $1.13 \mathrm{bar} / 77.3 \mathrm{~K}$ as a function of (a) BET surface area (blue symbols); (b) Langmuir surface area ( $\mathrm{N}_{2}$ sorption; red symbols); and (c) Langmuir surface area ( $\mathrm{H}_{2}$ sorption; green symbols). 

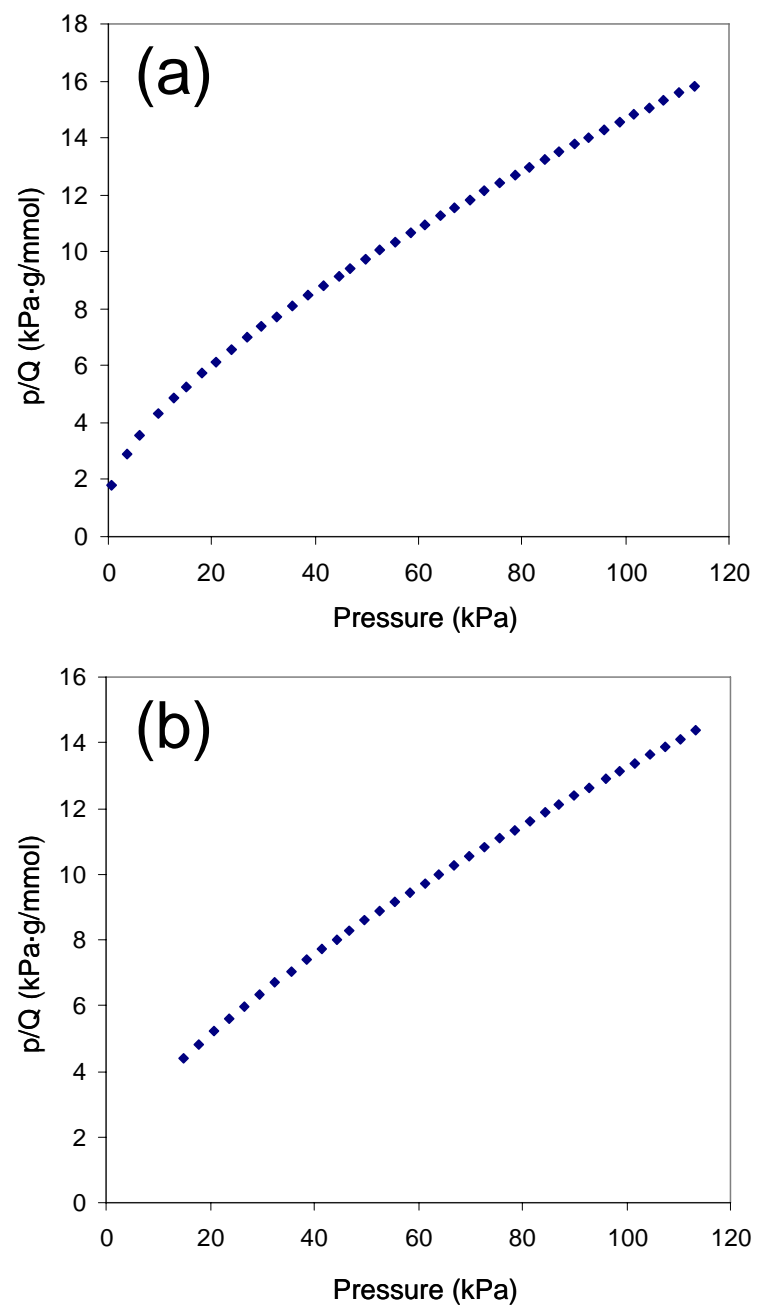

Figure S2. Langmuir plots ( $\mathrm{H}_{2}$ isotherm) for (a) HCPBVC; (b) Polymer 43. 


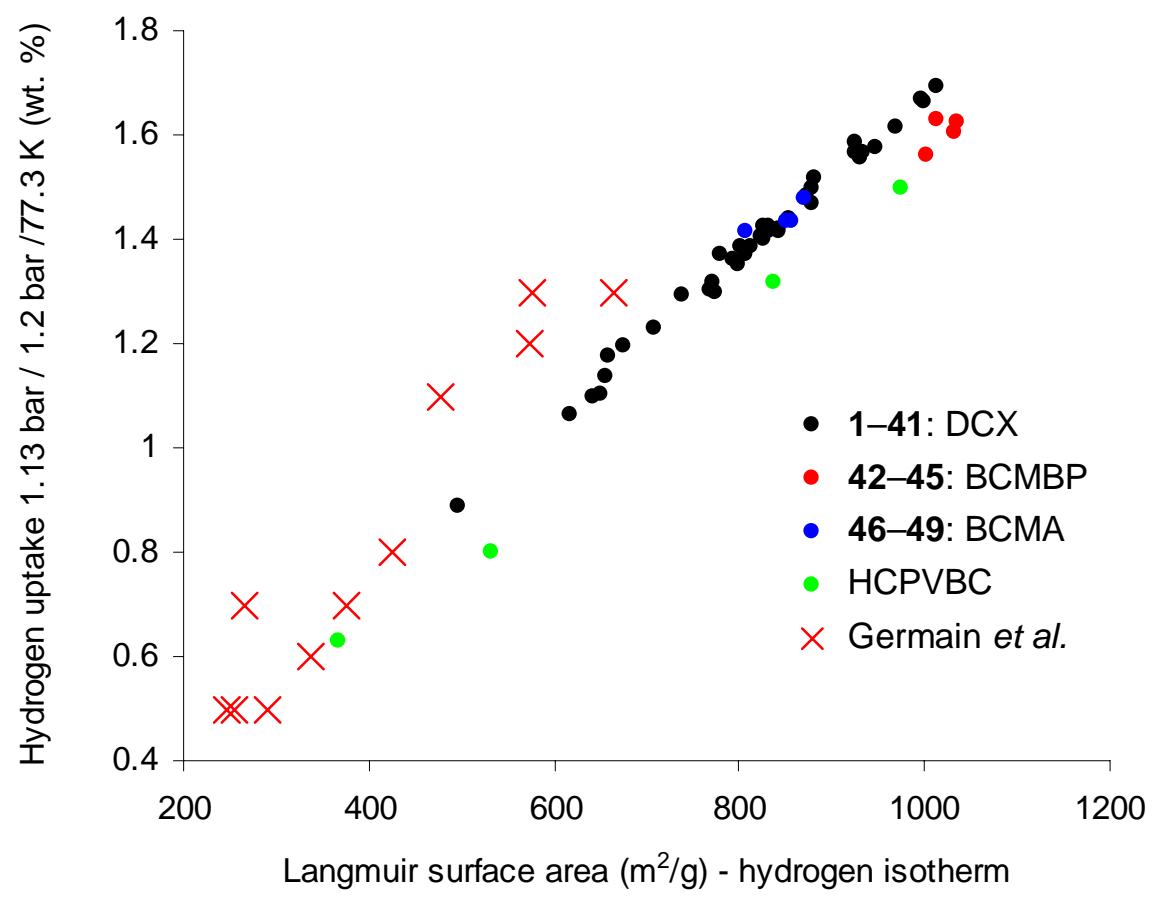

Figure S3. Plot of $\mathrm{H}_{2}$ uptake at 1.13 bar / $77.3 \mathrm{~K}$ for $\mathbf{1 - 4 9}$ as a function of Langmuir surface area (calculated from $\mathrm{H}_{2}$ sorption isotherms). Red crosses indicate data taken from Germain et al., (Chem. Mater. 2006, 18, 4430-4435) at a slightly higher $\mathrm{H}_{2}$ pressure of 1.2 bar. 


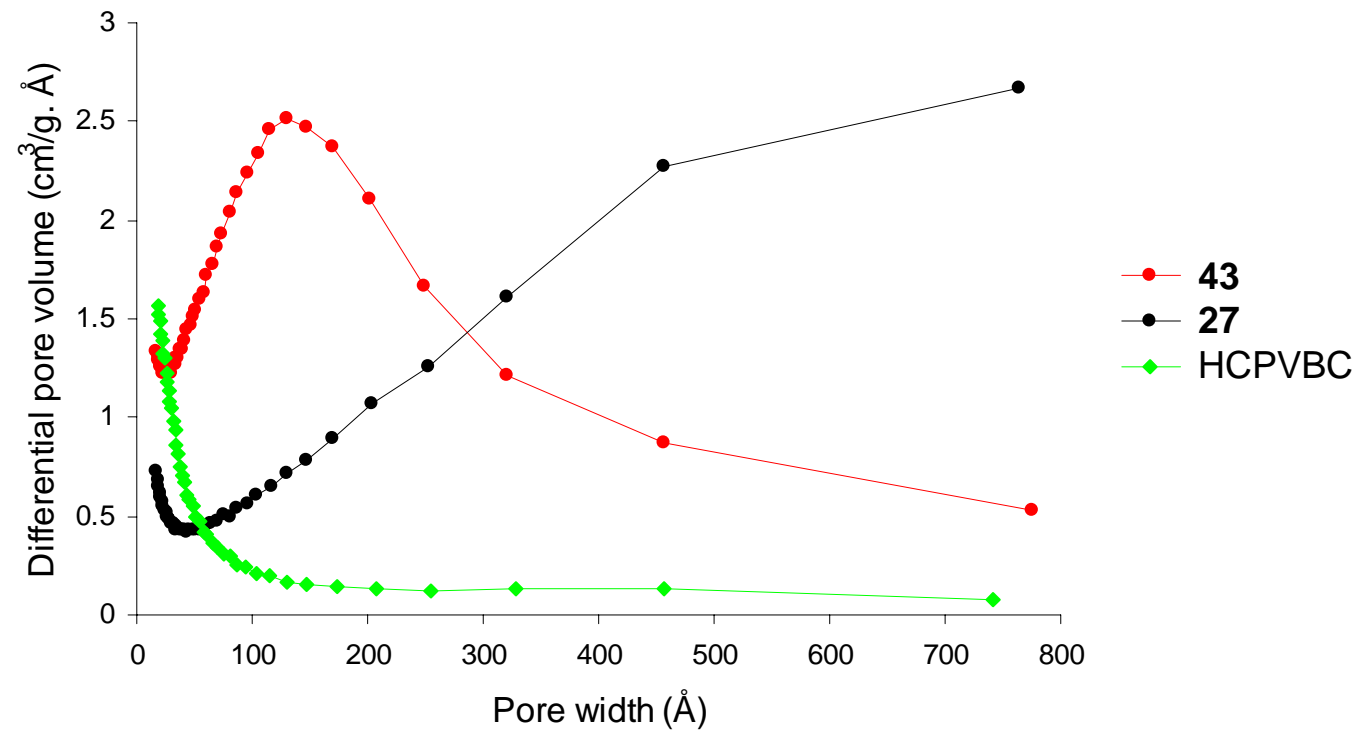

Figure S4. BJH pore size distributions for HCPVBC, 27, and 43 as calculated from the $\mathrm{N}_{2}$ adsorption isotherm at $77.3 \mathrm{~K}$. 

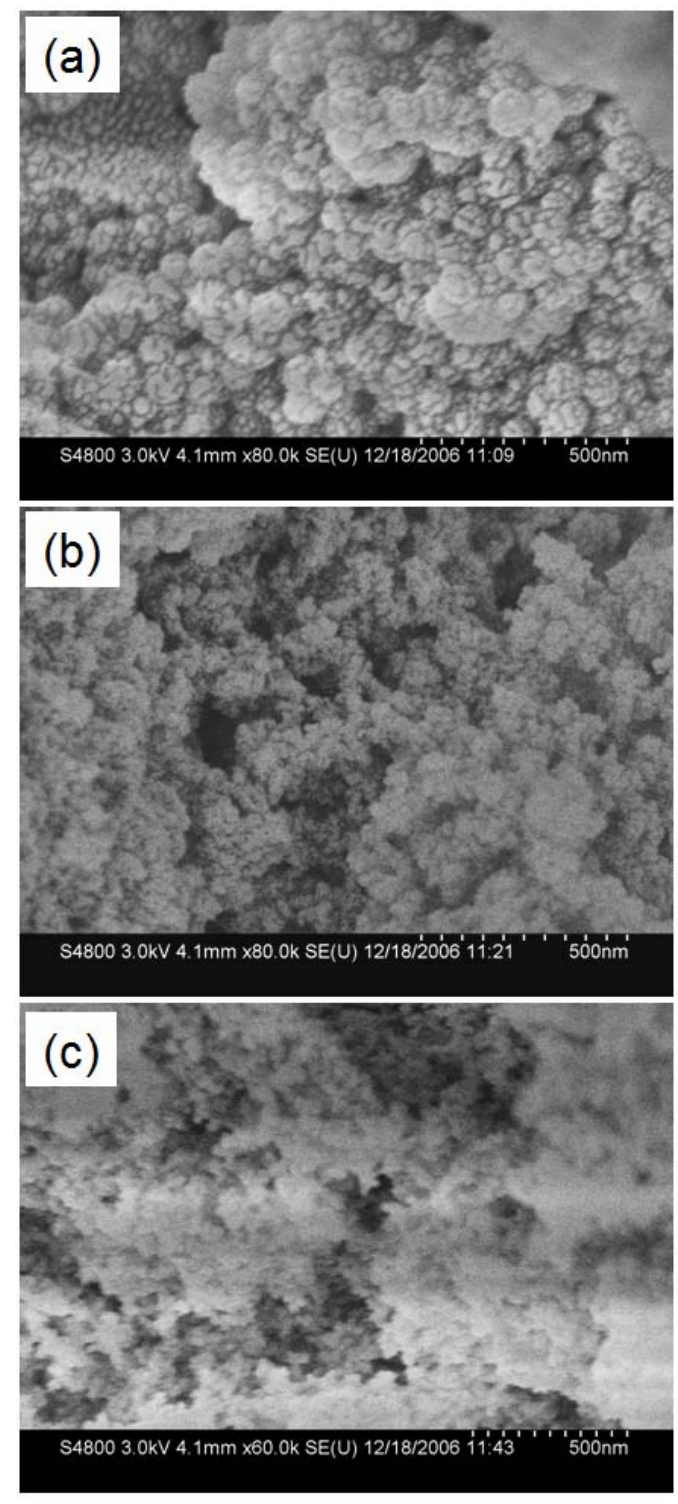

Figure S5. FE-SEM images for (a) HCPVBC; (b) 43; and (c) 27. The samples were sputter-coated with $\sim 1 \mathrm{~nm}$ gold before analysis - this is apparent in image (a) (the white "island" structures) but not discernable in (b) and (c). The HCPVBC sample exhibits a coarser, nodular morphology that is quite typical for suspension polymerized beads. HCPVBC does not show the fine mesoporous structure observed for 27 and 43. This stems from the different synthetic methods used to make these polymers - that is, hypercrosslinking of a single-phase gel versus precipitation polymerization; see main text for discussion. 


\section{DFT - cylindrical pore model}

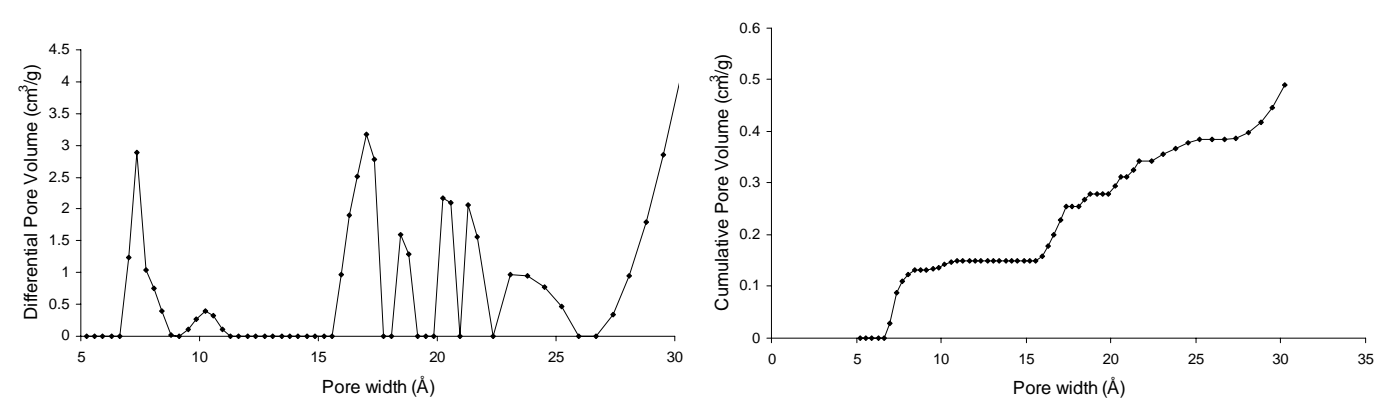

\section{DFT - slit pore model}
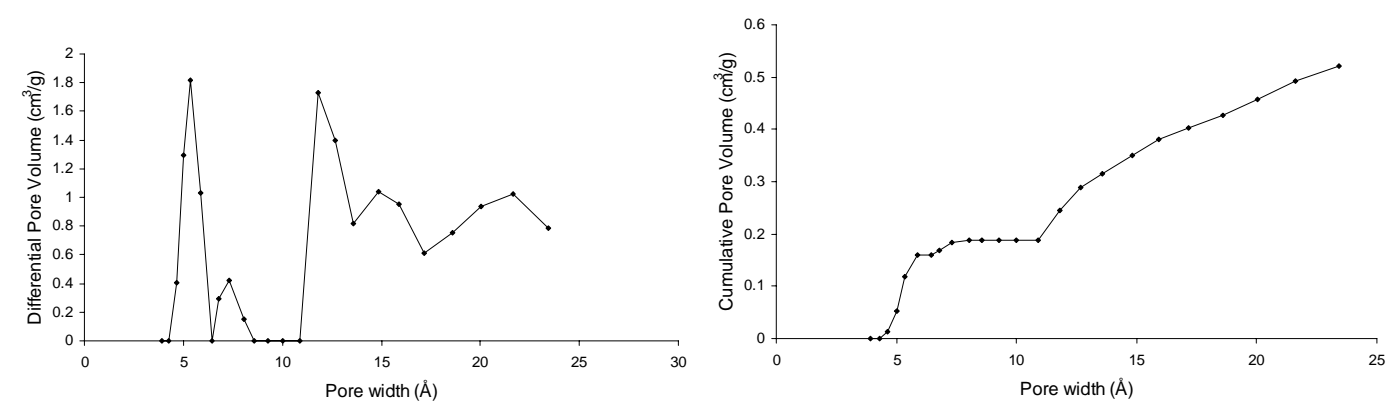

Figure S6. Micropore size distributions for 27 as calculated using DFT methods (cylindrical and slit pore models). 


\section{DFT - cylindrical pore model}

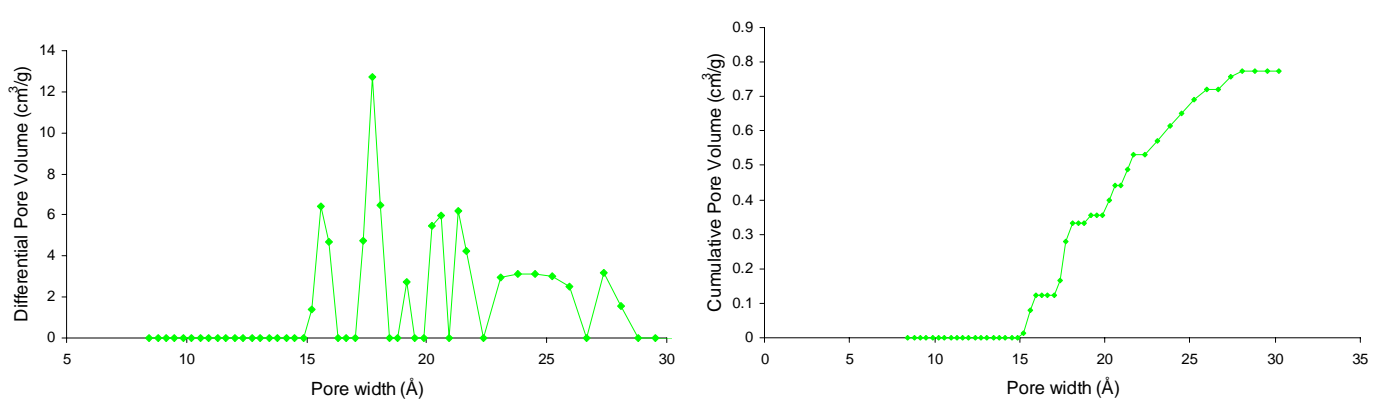

\section{DFT - slit pore model}
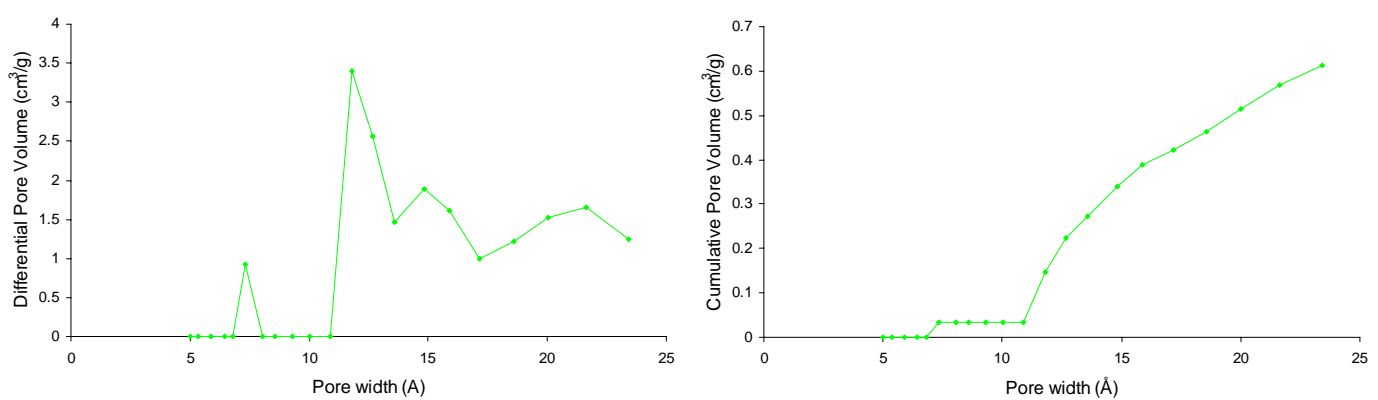

Figure s7. Micropore size distributions for HCPVBC as calculated using DFT methods (cylindrical and slit pore models). 


\section{DFT - cylindrical pore model}
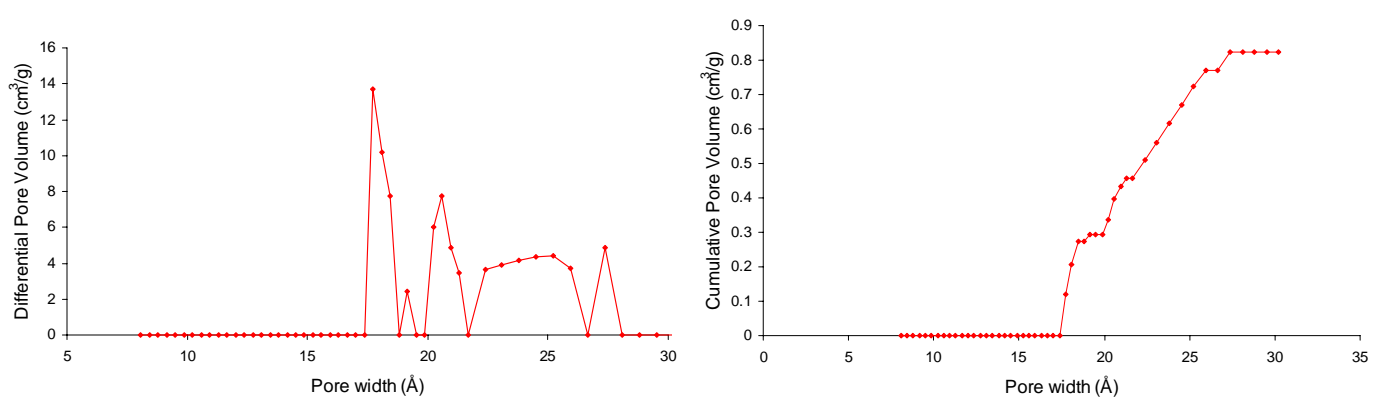

\section{DFT - slit pore model}
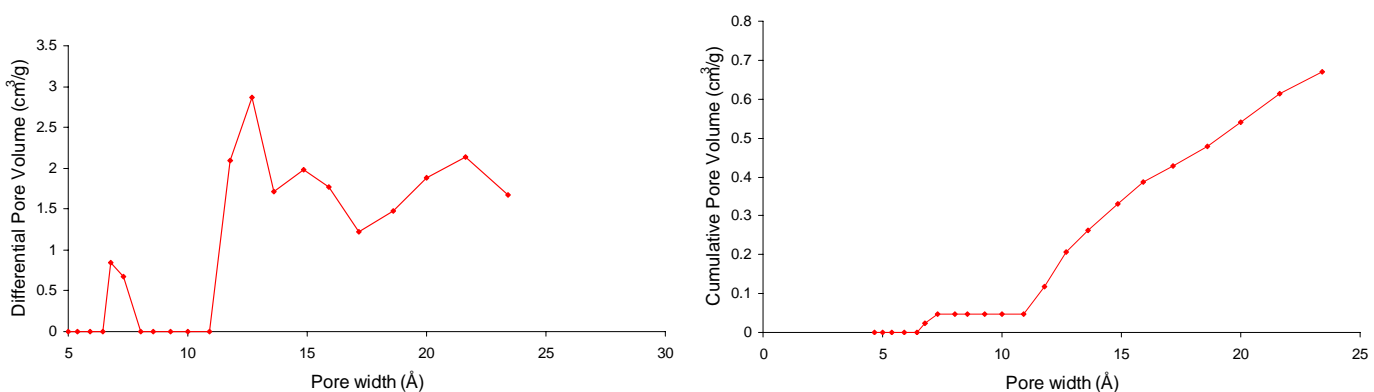

Figure S8. Micropore size distributions for $\mathbf{4 3}$ as calculated using DFT methods (cylindrical and slit pore models). 


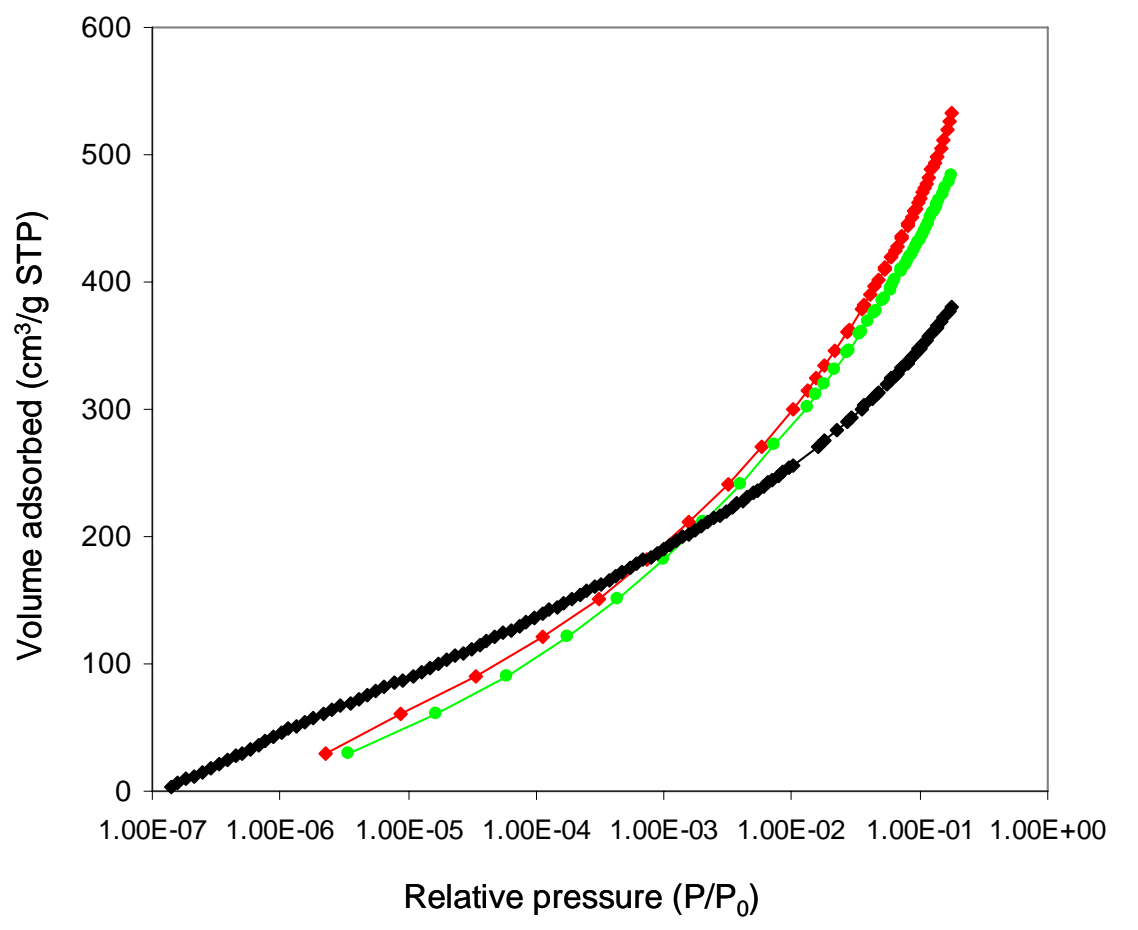

Figure 59. Semilogarithmic plot of $\mathrm{N}_{2}$ sorption at $77.3 \mathrm{~K}$ for HCPVBC (green symbols), 27 (black symbols) and $\mathbf{4 3}$ (red symbols). 


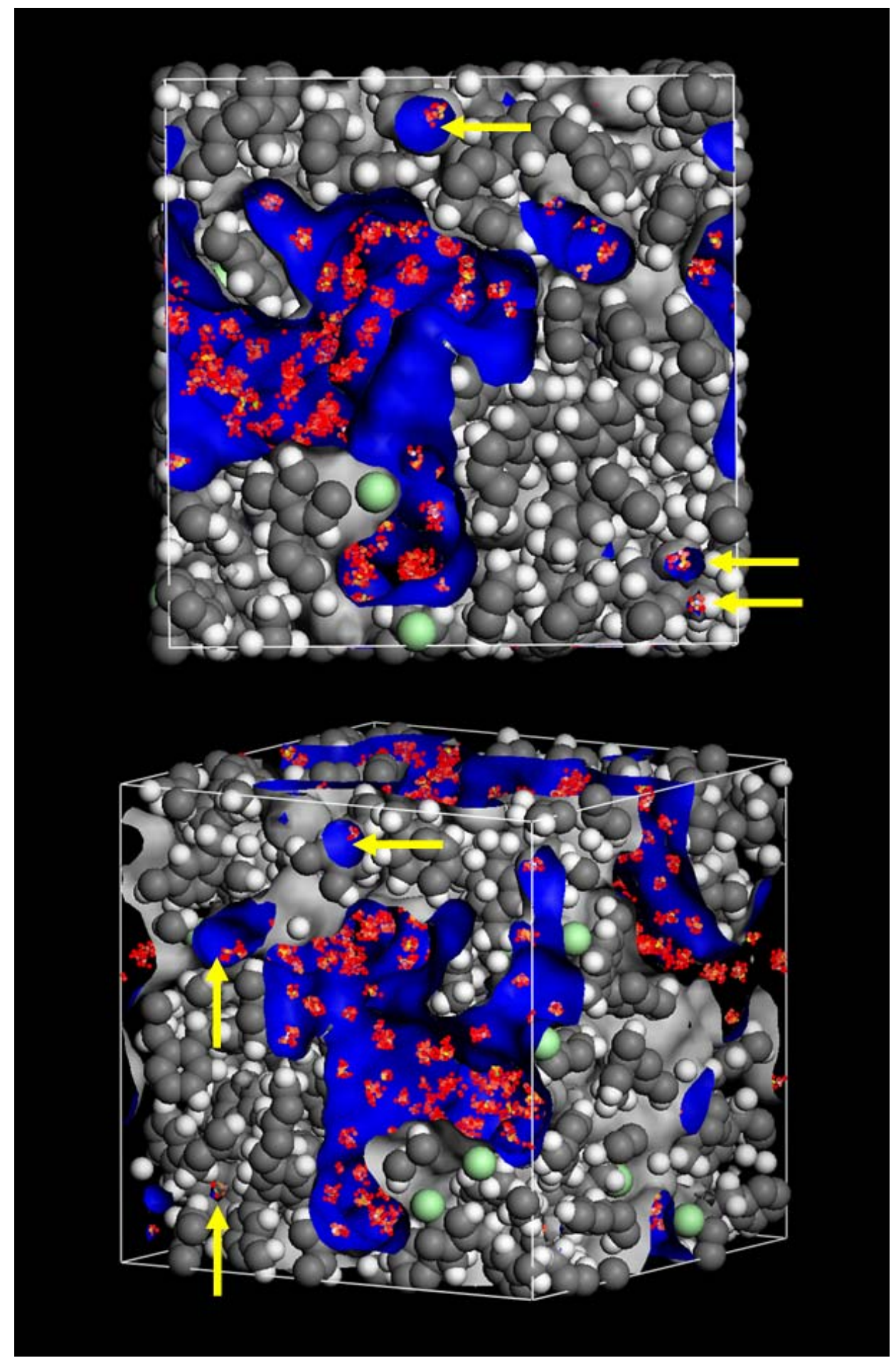

Figure S10. Snapshot of $\mathrm{H}_{2}$ sorption in the simulated pore structure at $1.1 \mathrm{bar} / 77 \mathrm{~K}$. The density field of the adsorbed $\mathrm{H}_{2}$ molecules is shown in red / orange. The yellow arrows indicate pockets of occluded free volume where $\mathrm{H}_{2}$ has been adsorbed in the simulation but would most likely be inaccessible to the sorbate in a real material. 


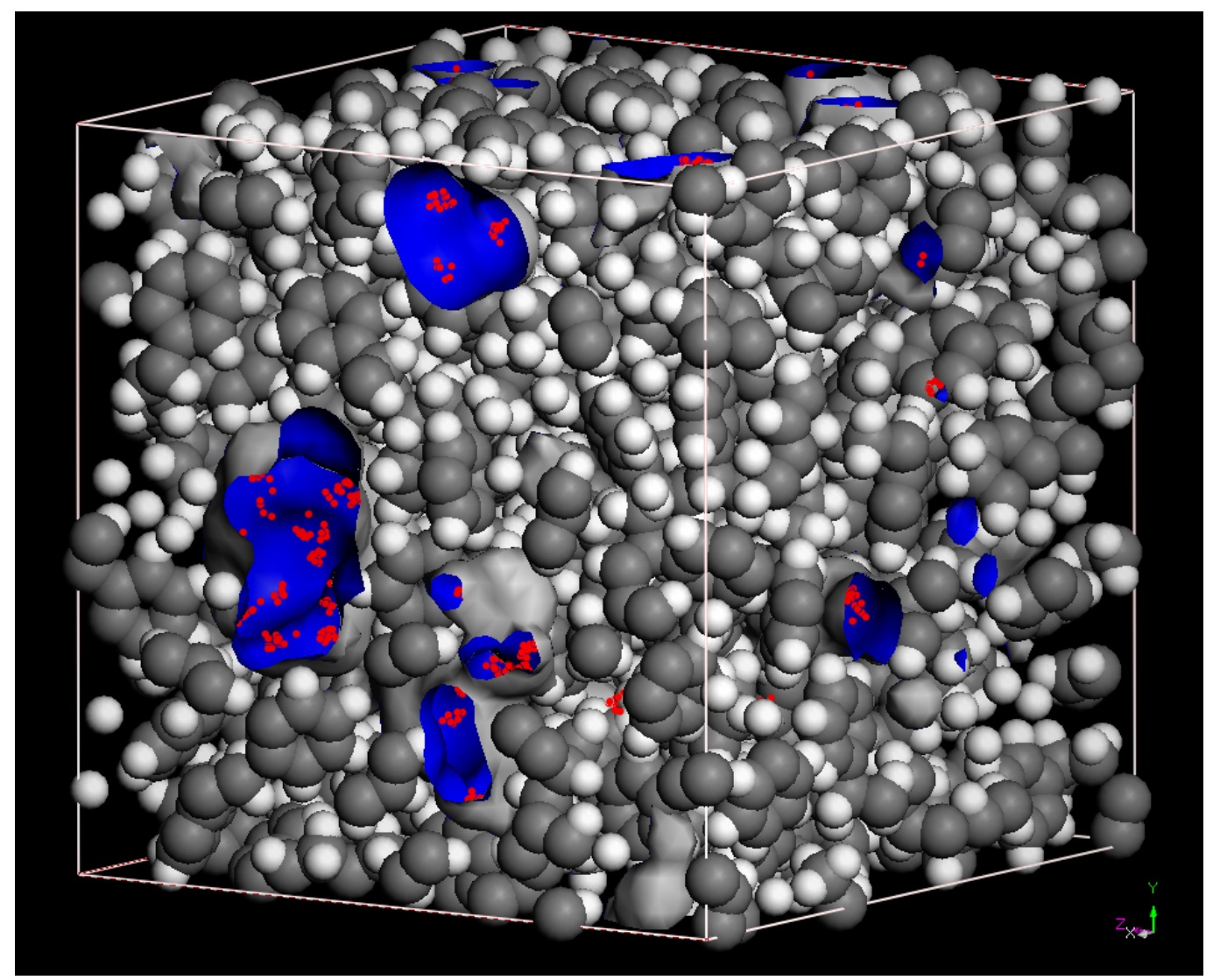

Figure S11. Atomistic simulation for amorphous polystyrene (10 chains each with 20 repeat units; bulk density $1.05 \mathrm{~g} / \mathrm{cm}^{3}$ ). A Connolly surface is shown in blue. A snapshot of $\mathrm{H}_{2}$ sorption in the structure at 1.1 bar / $77 \mathrm{~K}$ is superimposed; the density field of the adsorbed $\mathrm{H}_{2}$ molecules is shown in red / orange. The polymer is simulated to adsorb 1.23 wt. \% $\mathrm{H}_{2}$ under these conditions (see text). In reality, this occluded free volume would not be accessible to the sorbate molecules and the degree of sorption in non-porous atactic polystyrene would be expected to be negligible at $1.1 \mathrm{bar} / 77 \mathrm{~K}$. 


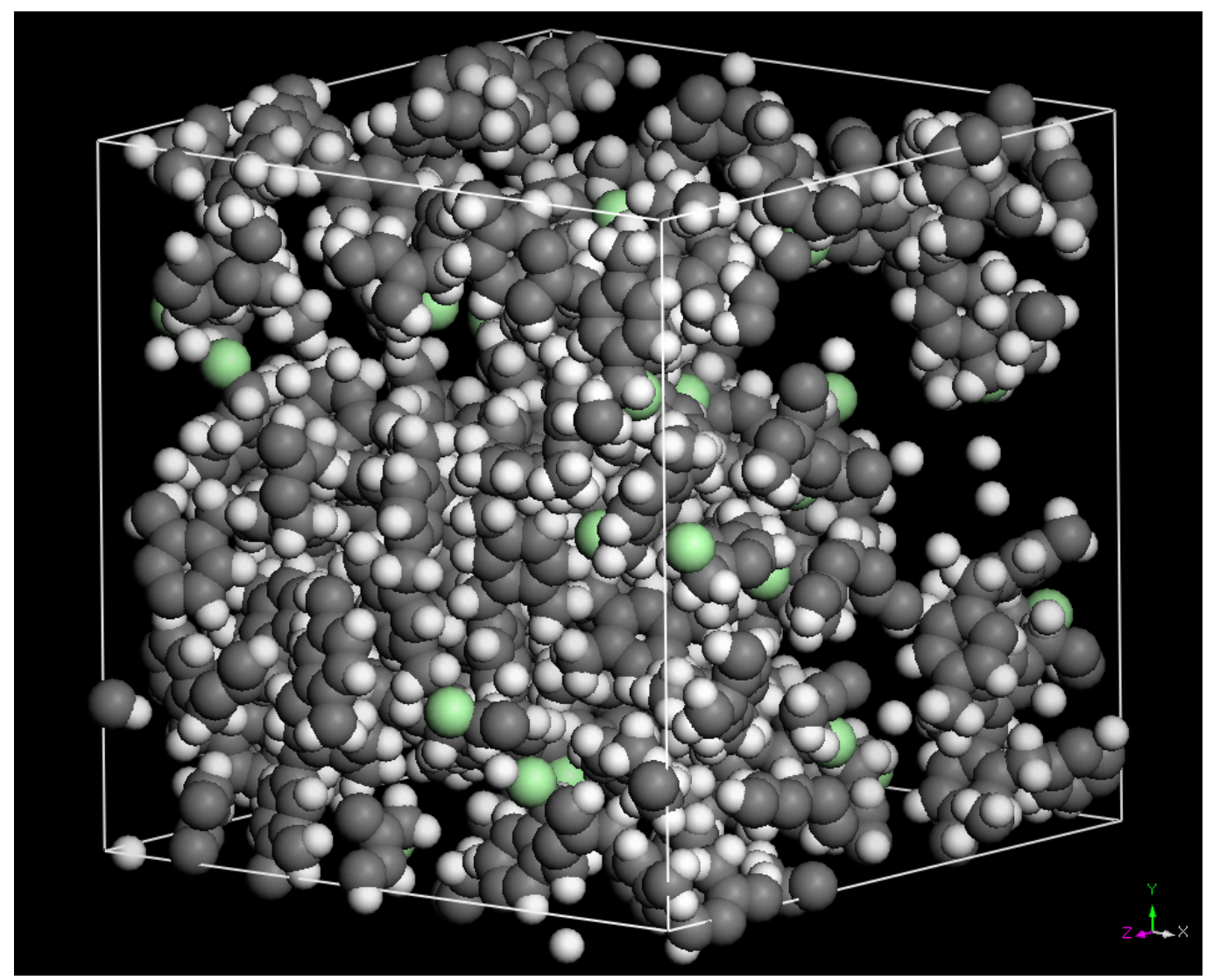

Figure S12. Simulated poly(p-DCX) network, Connolly surface area $=2519 \mathrm{~m}^{2} / \mathrm{g}$, simulated micropore volume $=0.551 \mathrm{~cm}^{3} / \mathrm{g}$. Dimension of simulation box (the “amorphous cell”) = 3.3175 nm. (Expansion of Fig. 10a.) 


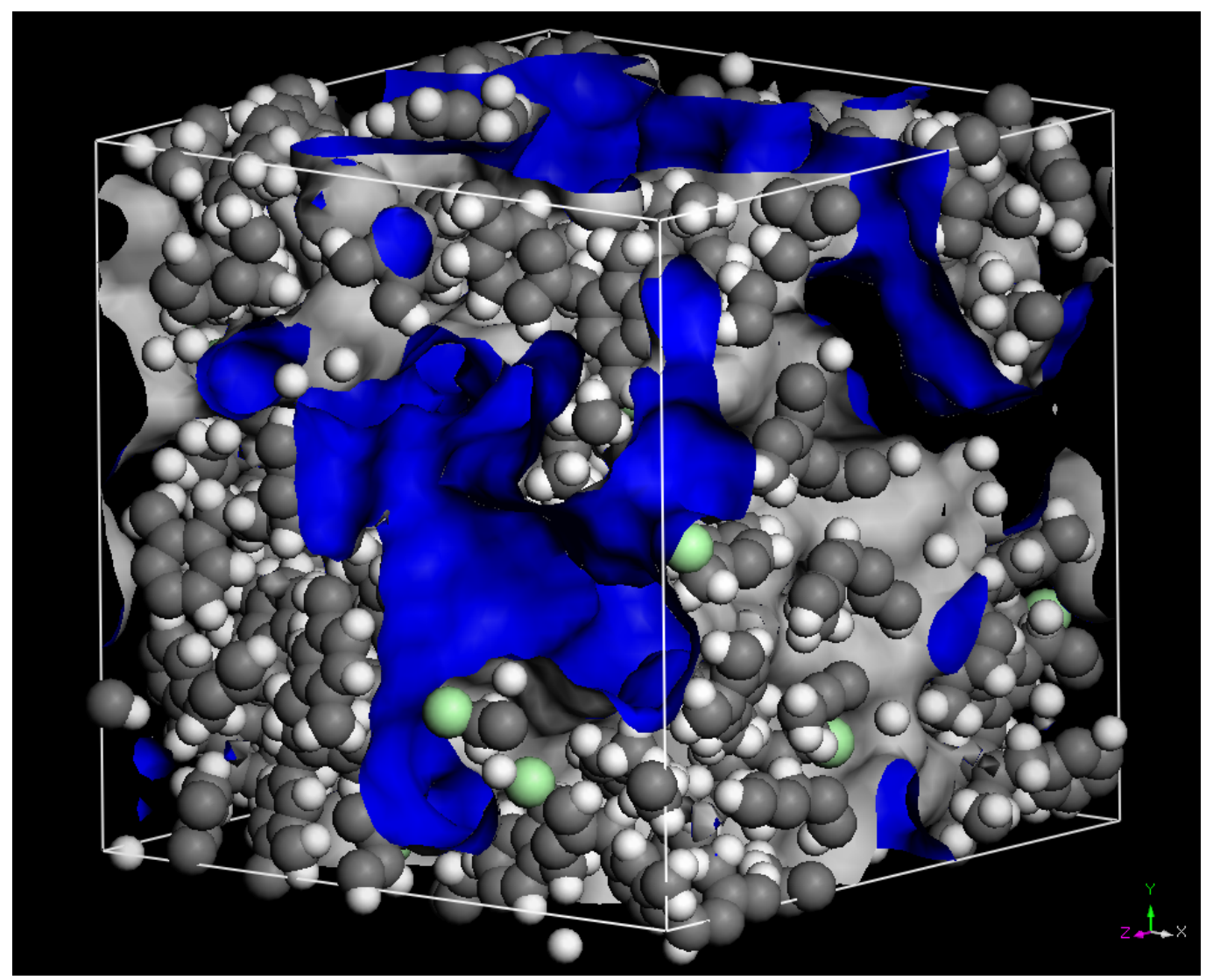

Figure S13. Simulated poly(p-DCX) network showing Connolly surface. Dimension of simulation box $=3.3175 \mathrm{~nm}$. 


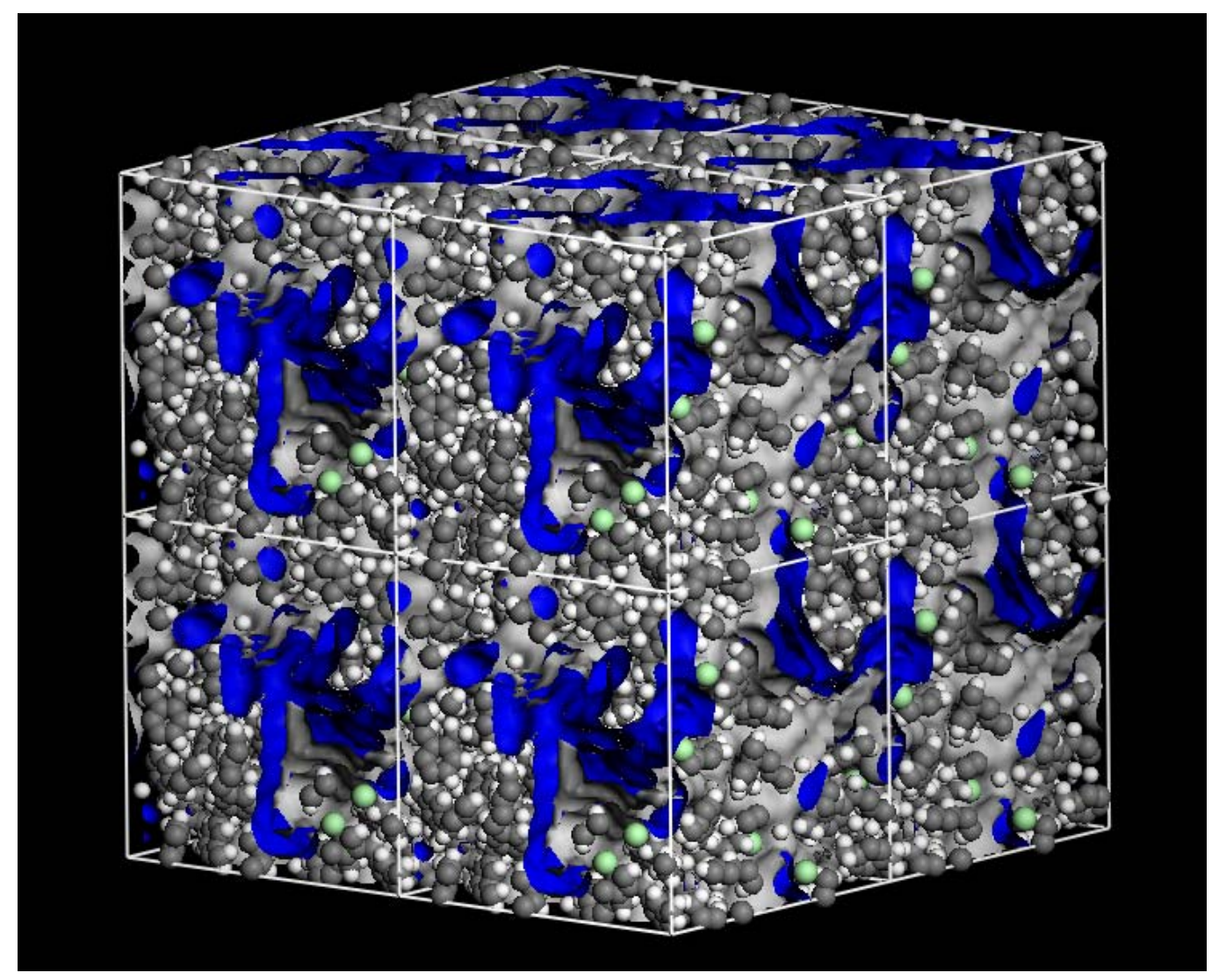

Figure S14. 3-Dimensional array of eight amorphous cells with periodic boundary conditions. A Connolly surface is shown in blue / gray. Each of the simulation boxes (8 are shown) has a dimension of 3.3175 nm. (Expansion of Fig. 10b.) 


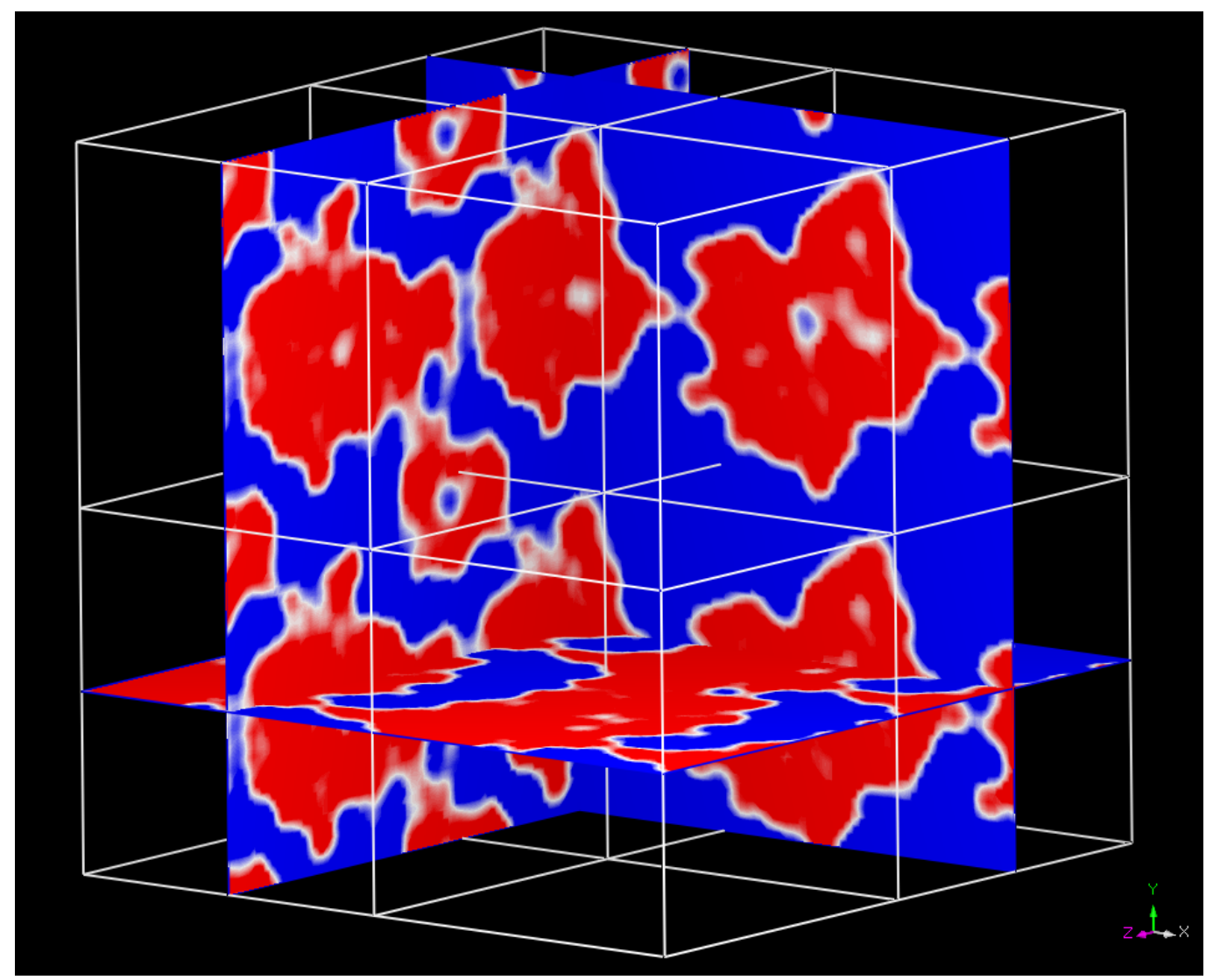

Figure S15. 2-Dimensional "slices" through the simulated pore structure. The occupied and unoccupied volume is shown in red and blue, respectively. Each of the simulation boxes (8 are shown) has a dimension of $3.3175 \mathrm{~nm}$. 


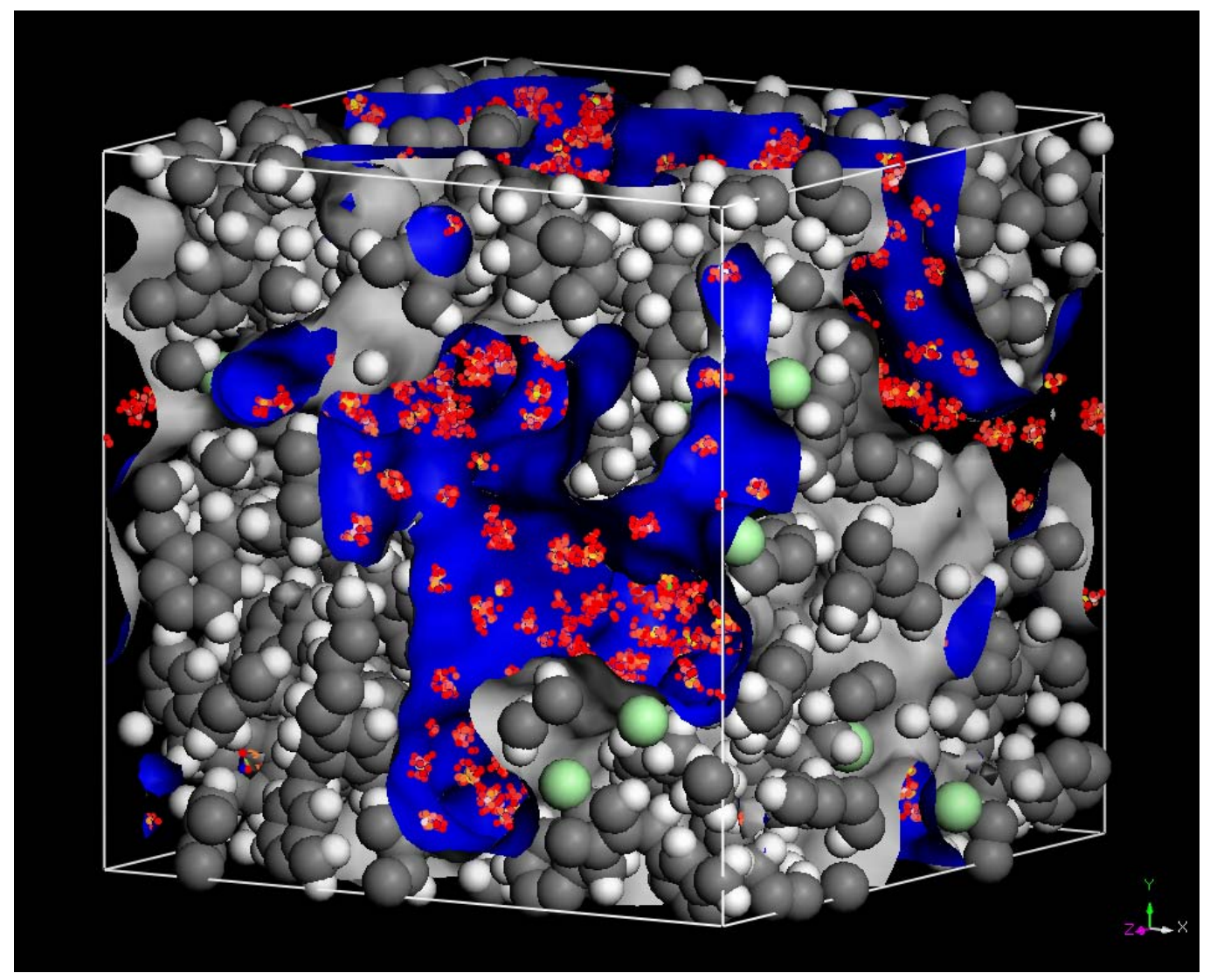

Figure S16. Snapshot of $\mathrm{H}_{2}$ sorption in the simulated pore structure at $1.1 \mathrm{bar} / 77 \mathrm{~K}$. The density field of the adsorbed $\mathrm{H}_{2}$ molecules is shown in red / orange (expansion of Fig. 10d; see also density map, Fig. S17). 


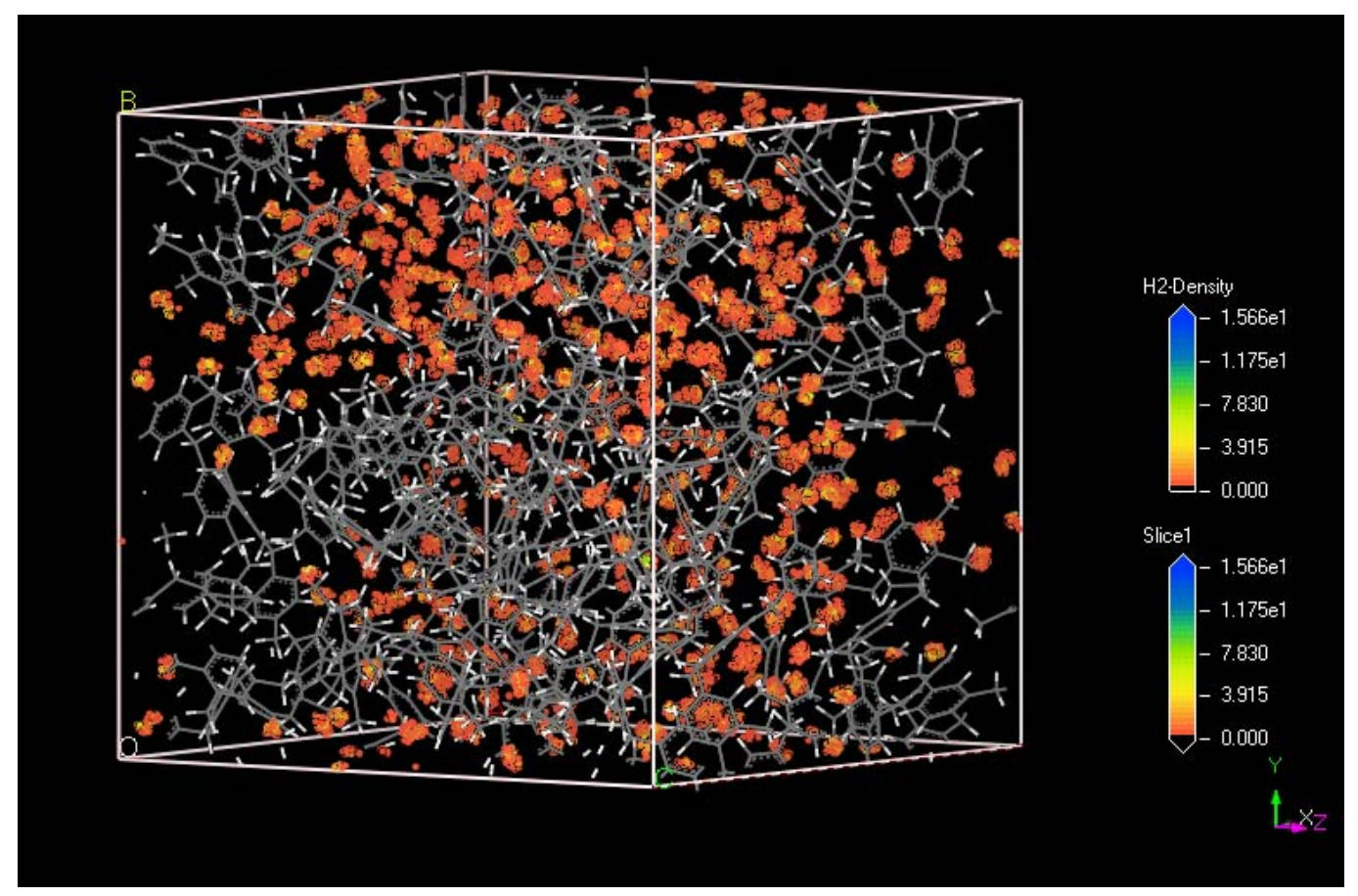

Figure S17. The density map of the adsorbed $\mathrm{H}_{2}$ molecules in the simulated microporous structure (Connolly surface removed). 